\title{
Digital Holographic setup for Measurement of Asymmetric Temperature Field and Tomographic Reconstruction
}

\author{
R. Doleček ${ }^{1,2}$, V. Kopecký ${ }^{2}$, P. Psota ${ }^{1,2}$, and V. Léd1 ${ }^{1,2}$ \\ ${ }^{1}$ TOPTEC,Institute of Plasma Physics, Sobotecka 1660, 51101 Turnov, Czech Republic \\ ${ }^{2}$ Departmentof measurements, Technical University of Liberec, Studentska 2, 46117 Liberec,Czech Republic
}

\begin{abstract}
This paper presents a digital holographic multidirectional interferometric method for measurement of periodic asymmetric temperature fields. This method uses a modified Twymann-Green setup having double sensitivity. The setup employs one precisely synchronized and triggered CCD camera with a measured coherent phenomenon. That allows a large number of the phenomenon's different projections capturing which are used for 3D tomographic reconstruction.
\end{abstract}

\section{Introduction}

Temperature field measurement is very important for thermodynamic research but it is not a simple discipline. The measurement requires contactless, non-invasive and very precise method which should be realized. There exist some methods for temperature fields measurement often based on the hot-wire anemometry [1]. These methods have one big disadvantage - measuring in a single-point. Therefore it is more suitable to use the method of digital holographic interferometry (DHI) [2] which is whole field and match the requirements mentioned above.

In the easiest case temperature fields are twodimensional or symmetrical ones and can be easily measured and evaluated [2, 4]. But asymmetrical temperature fields are more complicated. An examination of asymmetric temperature fields cannot be done without the use of a tomographic approach, which requires a large number of different projections $[2,7,8]$. In case of DHI it would be necessary to use many digital sensors in the measurement setup to obtain digital holograms for different view in directions. However, for a periodical coherent (self similar in each cycle) phenomenon the problem could be solved in a much smarter way - having only one digital sensor. The key point for such an approach is a precisely synchronized digital camera with the phenomenon drive. The camera is triggered in relation to the proper phase of the measured phenomenon. It allows us the measurement of very fast phenomena and the phenomenon evolution in time. Moreover with use of a rotation stage one can capture the area of interest from different directions in precisely defined relative phase of the phenomenon.
In DHI at least two digital holograms $h_{1}, h_{2}$ are captured representing two different states of the measured phenomenon. Digital holograms are interference patterns formed by a reference wave and an object wave passed through the phase object (measured temperature field). The maximum angle $\theta_{\max }$ between these two waves is related to the spatial frequency, which the detector can record without violating the Nyquist criterion. Thus $\theta_{\text {max }} \approx \frac{\lambda}{2 \Delta \varepsilon}$ where $\Delta \varepsilon \times \Delta \varepsilon$ is the pixel size of the detector

The numerical reconstruction is calculated by the multiplication of the digital hologram $h$ with complex amplitude of the reference wave $r$. Optical fields in the image plane $U$ are calculated by the Sommerfeld formula which describes the diffraction of a light wave at the hologram in distance $d$ far from the hologram. The Sommerfeld integral can be solved by Fresnel approximation which in discrete finite form can be written as:

$$
\begin{aligned}
& U(n, m)=\exp \left(j \pi \lambda d\left(\left(\frac{n}{N \Delta \varepsilon}\right)^{2}+\left(\frac{m}{M \Delta \eta}\right)^{2}\right)\right) \times \\
& \quad \sum_{k=1}^{N} \sum_{l=1}^{M} h(k \Delta \varepsilon, l \Delta \eta) r(k \Delta \varepsilon, l \Delta \eta) \times \\
& \quad \exp \left(\frac{j \pi}{\lambda d}\left((k \Delta \varepsilon)^{2}+(l \Delta \eta)^{2}\right)\right) \exp \left(-j 2 \pi\left(\frac{k n}{N}+\frac{l m}{M}\right)\right)
\end{aligned}
$$

where $j=\sqrt{-1}$ and $\lambda$ is the wavelength of the used light. $\Delta \varepsilon \times \Delta \eta$ is the detector pixel size and $N \times M$ is the number of pixels in perpendicular directions. Reconstructed intensity stands :

$I(n, m)=|U(n, m)|^{2}$.

From the two captured digital holograms complex wave fields $U_{1}(n, m)$ and $U_{2}(n, m)$ are reconstructed and phases are obtained by

This is an Open Access article distributed under the terms of the Creative Commons Attribution License 2.0, which permits unrestricted use, distribution, and reproduction in any medium, provided the original work is properly cited. 
$\varphi_{1}(n, m)=\arctan \frac{\operatorname{Im}\left\{U_{1}(n, m)\right\}}{\operatorname{Re}\left\{U_{1}(n, m)\right\}}$

$\varphi_{2}(n, m)=\arctan \frac{\operatorname{Im}\left\{U_{2}(n, m)\right\}}{\operatorname{Re}\left\{U_{2}(n, m)\right\}}$

where the index 1 denotes the first state of the temperature field and the index 2 belongs to second state of the field. The interference phase difference is modulo $2 \pi[2]$ and is given by

$\Delta \varphi=\begin{array}{cll}\varphi_{1}-\varphi_{2} & \text { if } & \varphi_{1} \geq \varphi_{2} \\ \varphi_{1}-\varphi_{2}+2 \pi & \text { if } & \varphi_{1}<\varphi_{2}\end{array}$

Let's assume the first hologram was recorded with refractive index field $n_{1}$ and second hologram with $n_{2}$. The relation between interference phase $\Delta \varphi$ and index of refraction $n$ can be written as:

$\Delta \varphi=\frac{2 \pi}{\lambda} \int_{L}\left(\Delta n-n_{\infty}\right) d l$

where $d l$ denotes the differential distance along the line $L, \Delta n=n_{1}-n_{2}$ and $n_{\infty}$ is refractive index of surrounding air. The Gladston-Dale equation relates refractive index $n$ to the density of gas $\rho$ and with the ideal gas equation one can obtain the temperature field $T$

$n-1=K P / R T$

where $K$ is the Gladstone-Dale constant, $P$ is the air pressure and $R$ is the gas constant.

The line integral transform defined in (5) is mathematically equivalent to Radon Transform RT[2]. According to theory of RT, the determination of asymmetric temperature field requires capturing of a large number of holographic interferograms from different direction. RT is mathematicaly described in polar coordinates:

$r(s, \theta)=\int_{-T}^{T} f(s \cos \theta-t \sin \theta, s \sin \theta+t \cos \theta) d t$

where $f(x, y)=f(t \cos \phi, t \sin \phi)$ is the temperature distribution, parameter $s$ signes shift distance of the line from the origin of coordinate system and $\theta$ is angle of projection. The equation (7) is simplified in $2 \mathrm{D}$ by treatment of the measurement volume cuts of plane slices which are stacked to 3D. The inverse RT reconstructs projections $r(s, \theta)$ back to the function $f(x, y)$. There exists alot of approaches and computer implementations which solve the inverseRT-Central slice theorem, Filtered back projection, Algebraic reconstruction techniques, etc.[7, 8].

\section{Experiments}

\subsection{Experimental setup}

For the measurement of transparent objects, so-called phase objects, a Mach-Zehnder holographic interferometer setup is usually used. Unfortunately, in our case the phase change is quite small thus the sensitivity of this interferometer is not sufficient. As was shown in previous experiments $[3,4]$ the use of interferometric setup (Fig. 1) based on a Twymann-Green interferometer is more suitable.In this setup the light travels through the phase object twice, which brings the double sensitivity of the setup. However, the setup is more complicated to adjust and there is more light lost.

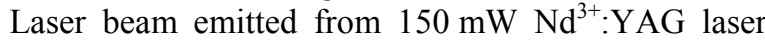
head is divided by polarizing beam splitter BS1. Both beams are further filtered by spatial filters SF and collimated by objectives CO. Beam no. 1 is reflected by mirror M2 to the beams splitter BS2. One part of the beam no. 1 goes directly through the beam splitter and objective $\mathrm{O}$ to $\mathrm{CCD}$ chip of camera. Second part is reflected to collimating objective. Beam no. 2 enters the beam splitter BS2. One part of the beam no. 2 is reflected in direction to the M2. Second part passes through the measured object. Mirror M3 is placed behind the object and it reflects the beam back through the object to the beam splitter BS2. There the beam undergoes second division and part of it is directed through the objective $\mathrm{O}$ to the CCD chip.



Fig. 1. DHI setup with double sensitivity (BS1-beam splitter, SF-spatial filter, CO-collimating objective, M-mirror, Oobjective, FG-functional generator, AMP-amplifier )

The object under investigation is Synthetic Jet SJ [5]. SJ are jets of fluid that are generated by pushing or pulling a fluid through $9 \mathrm{~mm}$ orifice by the interactions within the train of counter-rotatting vortex pairs. SJ are generated with frequency $15 \mathrm{~Hz}$ by one pairs of blowers. In the space in between the blowers there is placed a heating cartridge that heats up the air to increase the refractive index of the flux of air flowing from the orifice. The temperature of the heating cartridge is set to $150^{\circ} \mathrm{C}$ and it is controlled by a connected PID regulator. The generator of $\mathrm{SJ}$ is fixed on a rotation stage to get $2 \mathrm{D}$ holograms - projections from different viewing angles. The used camera is AVT Stingray F504 with resolution $2048 \times 2048$ pixels with size $3,45 \times 3,45 \mu \mathrm{m}$. The camera captures images of the area $50 \times 50 \mathrm{~mm}$ above the orifice. The exposure time is set to $20 \mu \mathrm{s}$ to get high-contrasted interference pattern image. 
The camera enables the frame rate of 6.5FPS for given resolution. To have a good temporal resolution it would be needed at least 300FPS for the given frequency $15 \mathrm{~Hz}$. However the coherence of the SJ phenomenon allows us to use the external trigger and to capture holograms in the same relative phase of the phenomenon in its every cycle. The camera capture time is synchronized with the driver of the SJ's generator. However for the frequency higher than camera feed rate the hologram is not captured after each synchronization signal but some cycles of the phenomenon are skipped. Therefore it is possible to measure very high frequency phenomena with use of a lower frame rate camera.

During the experiment, firstly, the reference hologram was captured for the switched off steady state SJ. In the next step the sequence of digital holograms for relative delays $0 \mathrm{~ms}, 2 \mathrm{~ms}, 4 \mathrm{~ms}, . . .66 \mathrm{~ms}$ (whole phenomenon period) was captured. The sequence consisted of 20 holograms for each delay. This process was repeated for angels $0^{\circ}, 10^{\circ}, \ldots, 160^{\circ}$.
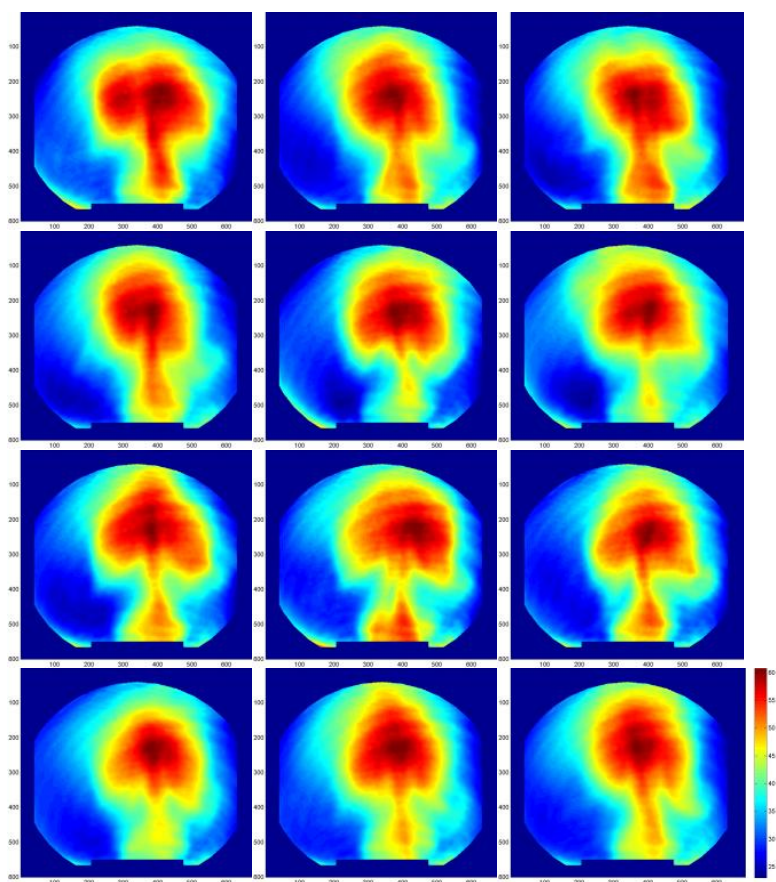

Fig. 2. Consequence of the precise synchronization. The sequence of evaluated temperature field distribution with angle of view $50^{\circ}$ and $16 \mathrm{~ms}$ relative delay.

\subsection{Thomographic reconstruction}

For the reconstruction of the digital holograms and data processing, semi-automatic software application in Matlab was developed. In the first step all recorded holograms were reconstructed by the equation (1) and interference phase differences fields were calculated by the formula (4) and the unwrapping Goldstein algorithm [6]. All phase fields corresponding to the same angle and the same time delay were averaged for elimination of random fluctuation. This way all data for 3D reconstruction were obtained. The reconstruction of $2 \mathrm{D}$ plane slices was realized by the method of filtered back projection. After the evaluation, many of such slices according to equation (6) were stacked to build the desire
3D temperature field. The calculation time for a processing of the digital holograms set corresponding to one phase of the phenomenon is about 30 minutes with use of $2.27 \mathrm{GHz}$ Intel i5 CPU.

\section{Results}

In Fig. 2 there is shown the consequence of the precise synchronization of the coherent phenomenon and the digital camera. The reconstructed 2D temperature fields correspond to different periods of the repeated phenomenon of SJ but with the same $16 \mathrm{~ms}$ relative time delay and $50^{\circ}$ angle of view. It can be easily seen the properties of the SJ puffs. It is also seen the temperature fields are nearly identical. The differences among them can be neglected because the thomographic reconstruction is performed from their average. Averaging eliminates random fluctuations and noise.

The evaluated dynamic temperature field is shown in Fig. 3. Holograms were captured with angle $50^{\circ}$ and with relative delays step $3.2 \mathrm{~ms}$. Results in Fig. 3 clearly represent dynamic development of the SJ.

The averaged data from different projections were input parameters of reconstruction algorithm. Finally resulting $3 \mathrm{D}$ volume data of temperature field generated by the SJ for $3,2 \mathrm{~ms}, 9,6 \mathrm{~ms}$ and $19,2 \mathrm{~ms}$ time delay calculated from 17 projections by method of filtered back projections are displayed in Fig. 4.
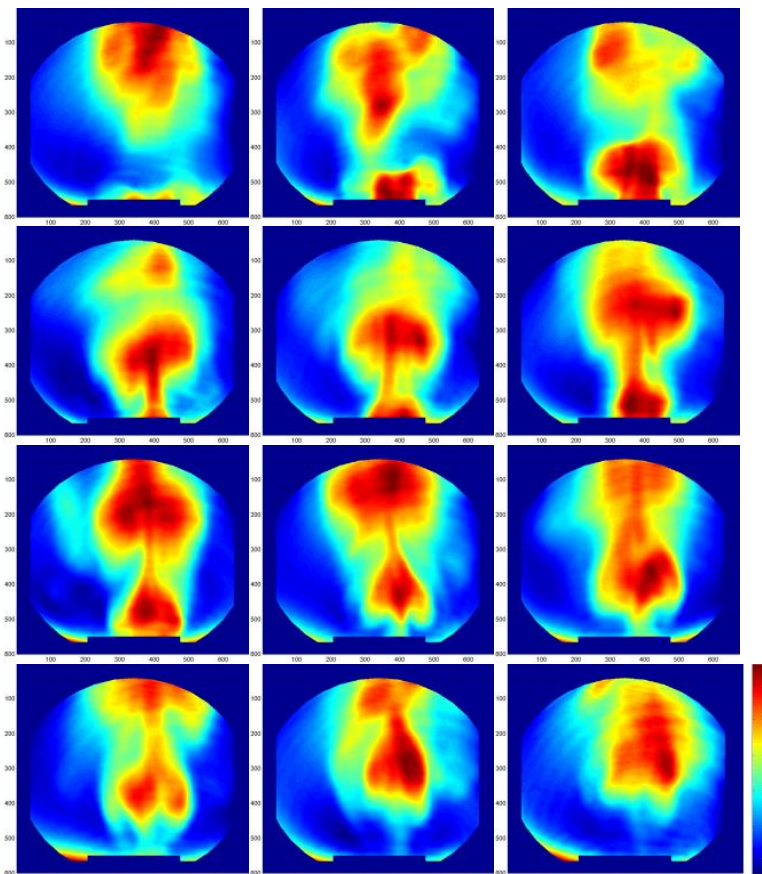

Fig. 3. The evaluated dynamic temperature fields with angle of view $50^{\circ}$ with the relative time delays $0 \mathrm{~ms}, 3,2 \mathrm{~ms}$, $6,4 \mathrm{~ms}, \ldots ., 38,4 \mathrm{~ms}$. 


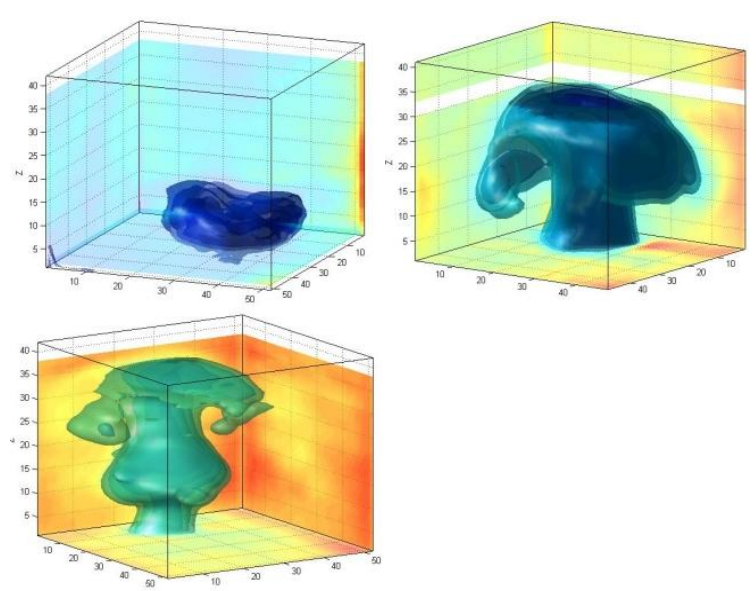

Fig. 4. The 3D volume data of the computed temperature field fo relative delay time $3,2 \mathrm{~ms}, 9,6 \mathrm{~ms}$ and $19,2 \mathrm{~ms}$.

\section{Conclusions}

The paper presents a simple and powerful DHI method for coherent and periodic phenomena measurement in asymmetric temperate fields. Instead of the generally used Mach-Zehnder's type of interferometer for transparent object measurement it is used a digital holographic interferometer which is based on the Twyman-Green's type of interferometer. The biggest advantage is its double sensitivity making possible a detection of very small phase changes in propagating rays through the inspected area. On the other hand the setup is more complicated to adjust and there is more energy lost.

The 3D measurement and reconstruction are based on tomographic approach. This approach requires capturing of a large number of holographic interferograms from different directions.For the data capturing, a precisely synchronized and triggered camera is used. This allows us the measuring of very high frequency coherent and periodic phenomena with use of a lower frame rate camera.

After brief introduction of DHI the hardware setup for asymmetric temperature field measurement is described in detail. The key features of the method as well as the synchronization and triggering settings of the digital camera and the phenomenon drive were described. Further the paper describes a basic data processing and the principle of tomographic reconstruction. The measuring method was successfully applied on a SJ temperature fields.

The measurement time necessary for capturing of all digital holograms was about 6 hours considering the manual manipulation with the rotation stage, manual settings delay times and holograms recording. In future the whole measuring process will be fully automatized.

\section{Acknowledgements}

This work was supported by the European Regional Development Fund and the Ministry of Education, Youth and Sports of the Czech Republic in the Project No. CZ.1.05/2.1.00/03.0079: Research Center for Special Optics and Optoelectronic Systems (TOPTEC) and by the Student grant scheme at the Technical University of Liberec.

\section{References}

1. StainbackP.C., NagabushanaK.A.: Review of hotwire anemometry techniques and the range of their applicability. In: Thermal Anemometry, Vol. FED167 ed. By ASME (ASME, New York 1993), pp 93-133

2. Kreis T.: Handbook of Holographic Interferometry:Optical and Digital Method (Berlin: Wiley, 2004)F. De Lillo, F. Cecconi, G. Lacorata, A. Vulpiani,EPL, 84 (2008)

3. Vít T., Lédl V., Identification of the temperature field in pulsative impinging flow. (2010) AIP Conference Proceedings, 1281, pp.135-138.

4. Doleček R., Lédl V., Kopecký V., Psota P., Václavík J., Vít T., Prospects of digital holographic inteferometry in heat transfer measurement, Experimental Fluid Mechanics 2009, Nov 25-27, (2009), Liberec Czech Republic.

5. Trávníček Z., and TesařV., Annular synthetic jet used for impinging flow mass-transfer, Int. J. Heat Mass Transfer, 46, 3291-3297, (2003)

6. VenemaT. M., SchmidtJ. D.: Optical phase unwrapping in the presence of branch points, 16 , 6985-6998 (2008).

7. Zhang Y. and Ruff G.A., Three-dimensional temperature measurements in enclosures by using multiview interferometric tomography, (1994) Meas. Sci. Technol. 5495 doi:10.1088/0957-0233/5/5/005

8. D. W. Sweeney and C. M. Vest, Reconstruction of Three-Dimensional Refractive Index Fields from Multidirectional Interferometric Data, Applied Optics, Vol. 12, Issue 11, pp. 2649-2664 (1973) 